\title{
Three Incomprehensible Binaries
}

\author{
Peter P. Eggleton \\ Institute of Geophysics and Planetary Physics, Lawrence Livermore \\ National Laboratory, Livermore CA94550, USA
}

\begin{abstract}
I describe three eclipsing double-lined binaries whose fundamental data (spectra, masses etc.) are totally out of line with theoretical concepts. Accepting the data at face value, they seem to imply that (i) a massive star (about $10-30 M_{\odot}$ ) in a moderately wide binary $(P \approx 50-250 \mathrm{~d})$ can eject almost its entire envelope to infinity, without shrinking its period substantially, (b) some formerly triple systems can become binaries through the merger of two of the three components, and (c) a red giant can be stimulated to lose half its mass in a stellar wind before reaching its Roche lobe.
\end{abstract}

\section{Introduction}

The three binaries that I wish to discuss have virtually nothing in common with each other, except for the fact that they severely challenge various conclusions of theoretical binary-star evolution that most of us would feel are rather well established. They are systems for which rather good data on masses, radii and spectral types exist, because they are double-lined eclipsing systems. Not one of the six components would appear exceptional if it were a single star, because for single stars the estimates of mass, radius and luminosity would be much less certain. The systems are V379 Cep (HR 7940; Gordon et al. 1998), OW Gem (HDE 258878; Griffin \& Duquennoy 1993) and V643 Ori (HD 294651; Imbert 1987). The data on these systems, taken from these references, is as follows: V379 Cep - (B2III + B2III $\left.1.9+2.9 M_{\odot} ; 5.2+7.4 R_{\odot} ; 99.7 \mathrm{~d}, e=0.15\right)$ OW Gem - (G8IIb + F2Ib-II; $\left.4.0+5.9 M_{\odot} ; 35+30 R_{\odot} ; 1259 \mathrm{~d}, e=0.52\right)$ V643 Ori - (K7III + K2III; $\left.2.0+3.4 M_{\odot} ; 22+16 R_{\odot} ; 52.4 \mathrm{~d}, e=0.014\right)$. The experimental uncertainties in these values are discussed in the references, although I have a few comments to add below.

Since these systems have nothing in common except their incomprehensibility, I discuss them separately. I should add however that I attempt to account for their anomalies. If you believe my attempts then maybe they are not incomprehensible after all, but I certainly do not put forward these attempts with confidence. 


\section{V379 Cep}

Until about 1990 this system was unremarkable, as an apparently normal B2III star with suspected radial-velocity variations that pointed to the possibility of binarity. Then it was discovered to eclipse, giving the $99.7 \mathrm{~d}$ period, and also to be double-lined. The two components have much the same spectrum, but one component is narrow-lined and the other broad-lined. From the eclipses the system clearly has a high inclination, and therefore the masses cannot be substantially greater than the spectroscopic values of $M \sin ^{3} i$ listed above. These masses are something like 3 to 5 times less than the masses one might reasonably expect. A rather tentative distance of 440 pc, explained by Gordon et al. (1998), leads to luminosites of order $5 \times 10^{3}$ and $10^{4} L_{\odot}$ for the less and more massive (sharp-lined and broad-lined) components respectively.

We might start by questioning the reality of the determination. Although there is a well-covered radial-velocity curve for the sharp-lined component, Gordon et al. (1998) obtained only four measurements for the broad-lined component, with very poor phase coverage. However even if we were to discount entirely the measurements of the broad-lined component, the single-lined massfunction of $1.04 M_{\odot}$, combined with the high inclination, would generate a severe problem at least for one component, whatever the mass ratio. But even if in addition to one good curve there is only one good point for the other star, provided that it is well away from conjunction the mass ratio should be reasonably determinable. This appears to be the case for this system. Although I am no expert in spectroscopy, my inspection of Fig. 2 of Gordon et al. (1998) convinces me that they are justified in their conclusion. The narrow line is so much narrower that it is not easy to see how it could interfere seriously with a determination of the centre of the broad line, and conversely. Perhaps we can hope that the velocity amplitude of the sharp-lined component has been underestimated by as much as $30 \%$, which would double the mass function. Then at near-equal masses we would have something like the masses expected for main-sequence stars of the right spectral type. But I believe that the procedure that they used (TODCOR) to disentangle the spectra allows for the fact that the two spectra influence each other. Here is certainly a case where one might hope that further data at still higher resolution would be important. My remaining discussion is based on the premise that the low masses are real.

The only kind of star which seems likely to produce a high luminosity at such a low mass, and for a substantial interval of time, is something with a horizontalbranch-like structure, i.e. a star with a helium-burning core, a hydrogen-burning shell, and a hydrogen-rich envelope. Actually the envelope might be hydrogenpoor compared with solar composition, but it should have some hydrogen in it to burn at the base of the envelope. Such an entity might have a nuclear lifetime of about $1 \mathrm{Myr}$, as compared with the lifetime for a comparably luminous uppermain-sequence star of perhaps 30-50 Myr. If one were to consider the possibility of a post-asymptotic-branch state, which seems the only real alternative, the lifetime would be a hundred times shorter.

If two components of a binary are both in a short-lived evolutionary state, as compared with either the previous main-sequence phase or the final compactremnant phase, then one would infer that they had closely equal masses to start with. This might seem unlikely a priori, although in fact a rather high 
proportion of initial close binaries do appear to have near-equal masses (Lucy \& Ricco 1979). But for binaries whose initial period puts them near the boundary of Case A and Case B, Nelson \& Eggleton (2001) found a substantial range of mass ratio in which there is the possibility of 'late catch-up', i.e. star 2 (by convention here the initially less massive component) lags in evolution prior to RLOF, but after gaining substantial mass during RLOF it accelerates and may catch up with, even overtake, star 1 . In such a system both stars may have helium-burning cores at the same time, although one would expect star 2 to be several times (perhaps 10 times) the mass of star 1 at this point.

This kind of evolution, which Nelson \& Eggleton (2001) referred to as subCase AL, is illustrated in Fig. 1 with an example whose initial parameters are $\left(7+6.3 M_{\odot} ; 3 \mathrm{~d}\right)$. Panel (a) shows the theoretical HRD, and panel (b) the variation of radius with mass for both components as well as for the Roche lobes. The run was terminated at the point where star 2 reached its own Roche lobe; coincidentally star 2 had just ignited helium in its core, and by a further coincidence star 2 was also in the middle of the Cepheid strip. At this point star 1 had also ignited helium in its core, as evidenced by the small hook at the end of its computed evolution in the bottom left-hand corner of the HRD. The parameters at this stage were $\left(1.1+12.2 M_{\odot}, 100 \mathrm{~d}\right)$. If one were to ignore the reverse RLOF that should now take place, and instead allow both components to evolve as if they were single, star 1 would climb slowly up and then to the right in the HRD, becoming a largely helium red (super)giant before collapsing rapidly to a $\mathrm{C} / \mathrm{O}$ white dwarf, while star 2 would evolve much more rapidly, and would presumably become a supernova as a red supergiant well before star 1 became a white dwarf.

Of course we cannot ignore the reverse RLOF; but it appears to me that we have to demand a rather unconventional type of behaviour at this point. I believe that conventional wisdom would say that the reverse RLOF would be a common-envelope event, because of the extreme mass ratio, and the system would rapidly make the transition to a very close binary consisting of the core of star 2 (which is $\sim 2.8 M_{\odot}$ ) and star 1 . However we have to require that this did not happen, since the present period is not short at all. Instead, I suggest that the star 2 must have lost most of its envelope to infinity, but a proportion $(\sim 8 \%)$ would have to be accreted by star 1 in order to bring up its mass to what is observed, a very small proportion $(\sim 1 \%)$ would have to be retained, and only a modest amount of angular momentum would have been lost to infinity along with the envelope so that the system maintained much the same orbit as immediately before. The extra envelope which I suppose was accreted by star 1 may help it to produce the luminosity which it now has, by hydrogen-burning in a shell.

Nelson \& Eggleton (2001) computed a large number of Case A binaries, as well as a small number of Case B binaries with slightly longer periods. SubCase $\mathrm{AL}$, and the analogous subCase $\mathrm{BL}$, were quite common in the mass range that we require, and with periods fairly near the A/B borderline: see Fig. 9 of Nelson \& Eggleton (2001). The initial mass ratio was not particularly critical. The extensive RLOF, assumed conservative, normally makes the period increase by a factor of $\sim 20-40$, putting it fairly close to the current period of V379 Cep, so that it must have remained roughly constant in our hypothetical envelope ejection event. 
(a) Theoretical HRD

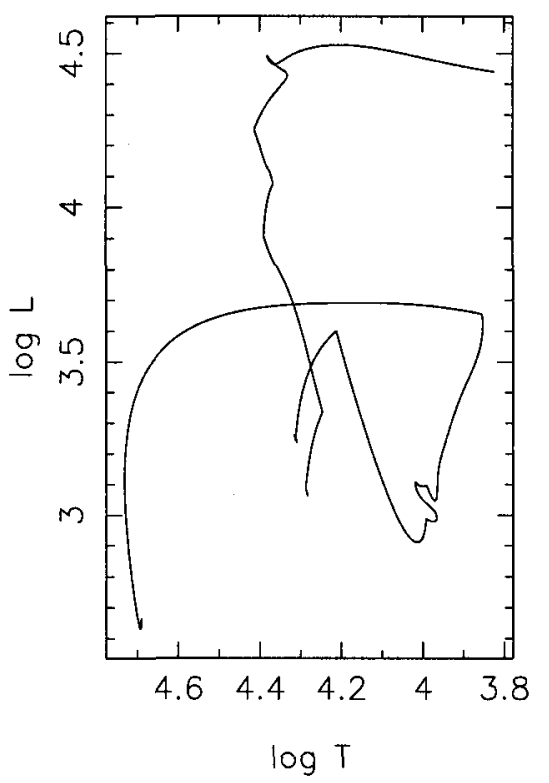

(b) $\log R$ vs $M$

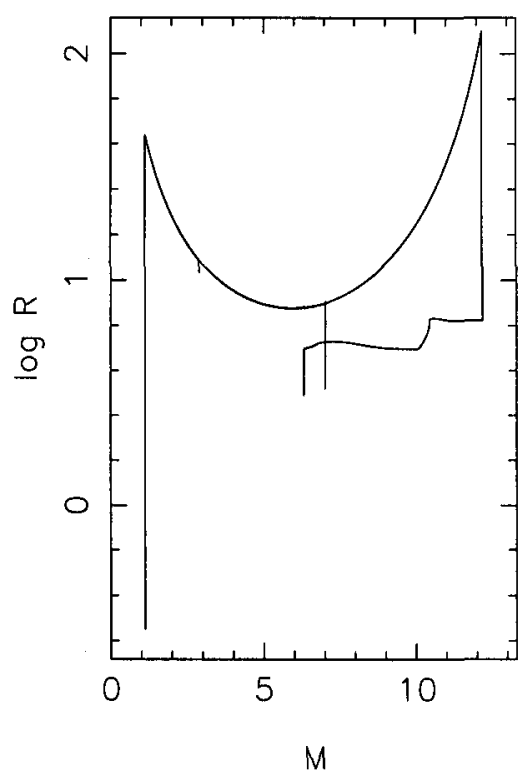

Figure 1. Possible evolution of the precursor of V379 Cep from the ZAMS to the point where star 2 fills its Roche lobe (reverse RLOF). (a) The theoretical HRD: the stars start from the ZAMS with masses $(7+$ $6.3 M_{\odot}$ ) at points just below the middle of the plot. The initial period was $3 \mathrm{~d}$. When star 1, the initially more massive, was a helium-burning remnant (lower left), star 2 almost simultaneously ignited helium in its core and filled its Roche lobe (upper right). The period by then was $100 \mathrm{~d}$. (b) The variation of stellar and Roche-lobe radius with mass. The stars start again near the middle of the plot, and end respectively at the lower left and upper right, with star 2 having just reached its Roche lobe. The evolution was assumed conservative up to this point. 
One might ask: if the reverse RLOF is highly non-conservative of mass, as we require, then is it reasonable to suppose that the earlier forward RLOF was conservative? I would argue that the difference is the orbital period. The much larger period for the reverse RLOF means that star 2 has a distended supergiant envelope, instead of the fairly compact envelope of a near-main-sequence star.

Single supergiants of $z 30 M_{\odot}$ are thought to blow off their entire envelopes in a P Cygni phase near the Humphreys-Davidson (1979) limit, a line at high luminosity with a shallow slope downwards to the right across the top of the HRD. It is thought that the envelope becomes unstable when it is large and close to the Eddington limit. A star of $\sim 12 M_{\odot}$ is well below this limit; but I conjecture that the extra perturbation due either to the fact that it is close to its Roche lobe, or to the fact that tidal friction will make it rotate much more rapidly than if it were single, might be enough to ensure a $P$ Cygni instability despite the fact that $L / M$ is somewhat further from the Eddington limit. It may be a misnomer to think of this ejection as a form of reverse RLOF, because possibly it happens even before, though not far before, RLOF would have been reached, and might prevent RLOF entirely.

Gordon et al. (1998) found V379 Cep to be in a relatively empty bubble at the edge of a star-forming region. They suggested that this bubble might be evidence of a former wind from the system. My suggestion is roughly consistent with this.

In answer to the question, "why is the orbit of V379 Cep eccentric?" I suggest that this is because the envelope ejection was intermittent, with short bursts of rapid mass loss, somewhat as seen in P Cygni stars. If a modest amount of mass is lost on something like the orbital timescale, in the last stages of envelope ejection, this might account for the modest eccentricity.

I have written elsewhere (Eggleton 2002) about this and three other systems - $v$ Sgr, PSR 0045-7319, and V2174 Cyg - which though very different from each other all seem to require much mass loss without serious orbital shrinkage. Their present periods are in the range 50-225 d, and I infer masses immediately before the hypothetical envelope ejection to be in the range $10-25 M_{\odot}$. I suggest that envelope ejection, rather than common-envelope evolution, may be the typical response of a binary component in this mass and period range to evolution close to its Roche limit.

Chochol (page 143), and Mayer etal. (2001) have described V505 Mon, a fairly massive eclipsing binary with a $54 \mathrm{~d}$ period containing (a) two stars that are not very close to filling their Roche lobes, and (b) an obscuring disc of gas around (probably) the more massive one. The masses and radii in this interacting system are much less securely determined than in the non-interacting system V379 Cep, but a plausible model is that the currently less massive component has lost, and is losing, much mass while the other is accreting only a proportion of the mass. I suggest that the less massive component here is near the end of the hypothesised envelope ejection phase; the orbital period is just in the range that I have already suggested. 


\section{OW Gem}

OW Gem consists of two yellow/orange supergiants, which we loosely refer to as red giants. There are several wide, detached binaries of red giants that conform to the following expectation from theoretical models: their masses are closely similar. Because most of a star's active life, $60-90 \%$ of it, is spent on the main sequence, and because the length of this active life depends strongly on the initial mass, two components of a binary that are both red giants should differ in mass by no more than about $15 \%$, except that at masses of $\sim 2-3 M_{\odot}$ the core helium-burning phase can be relatively long and might allow a mass difference of $\sim 20 \%$. One can certainly rule out the observed mass ratio $q \sim 1.5$ (or $q \sim 0.67$ ) by Griffin \& Duquennoy (1993) for OW Gem.

As before, we can start by speculating that there is something wrong with the observations, e.g. that one component at least is some anomalous star masquerading as a more normal star. For example, there are F-type shell stars where what is essentially a normal main-sequence star is masked by a cool lowgravity shell, typically acquired by accretion from a companion. I would only say that (a) one of the most experienced and distinguished spectroscopists on the planet did not note any such abnormality, and (b) such a masquerade would raise as many problems as it answers. The two components, though large, are very far inside their Roche lobes in this wide (3.4 yr) binary, even supposing that 'Roche lobe' can be defined meaningfully at periastron in such an eccentric orbit.

One can contemplate that there might have been some kind of mass loss or exchange, presumably when one or other component was temporarily much larger in the past. I do not have the space to go over every conceivable possibility, but I think that the long period, the eccentricity, and the normality (taken separately) of the components argues against this.

When one is at one's wits' end, one cries, "Triple star." In this case, I believe $\mathrm{OW}$ Gem is a former triple, with the $\mathrm{F}$ supergiant being the merged remnant of a close sub-binary, whose parameters might have been $\sim\left(4+2 M_{\odot} ; 2 \mathrm{~d}\right)$. The essence of my case is that (a) observationally, triple stars of the right character are not uncommon, and (b) theoretically, mergers of short-period binaries should also be quite common.

Among the 500 brightest stars, from the Bright Star Catalogue (Hoffleit \& Jaschek 1983) and the Catalogue of Spectroscopic Binaries (Batten et al. 1989), it seems to be easy to identify at least nine triples with longer period less than $30 \mathrm{yr}$. The shortest longer period is $33 \mathrm{~d}$ ( $\lambda$ Tau), and there are a few more with period shorter than $3.4 \mathrm{yr}$. Nine, even 500 , is a small number to do statistics on, and one can suppose that triple stars are somewhat over-represented in a magnitude-limited sample, but the required kind of system does not appear to be ridiculously rare. At the same time, recognising triplicity is probably harder than recognising duplicity, so there may be a few more such systems to be discovered.

Nelson \& Eggleton (2001), in their study of 5550 theoretical close binaries, identified 8 subclasses of Case A evolution, and also some similar classes in marginal Case $B$ evolution. At least three of these seem destined to end in a merger. They are: 
Case AD - dynamic-timescale RLOF. When the initial mass ratio is rather large, RLOF, supposed conservative initially, makes the orbit shrink rapidly and may cause the rate of mass transfer to spiral up until there is something like common-envelope evolution. How large a mass ratio will do this is unclear, but the critical value might be $q_{0} \sim 3-4$.

Case AR - rapid (thermal-timescale) evolution to contact. For more moderate mass ratios (perhaps $q_{0} \sim 1.5-3$ ), the expansion of the gainer in response to accretion, combined with the contraction of the orbit, brings the binary to contact very soon after RLOF begins, and long before $q$ is reduced to unity. Further evolution may be as a contact binary, a W UMa system if its mass is not large. Although the long-term evolution of these is not clear, the fact that several of them have quite extreme mass ratios (10:1, or more), and that almost all have rather large mass ratios (2:1 or more), strongly suggests that most probably they evolve slowly towards a merger, driven either by nuclear-timescale expansion or by magnetic braking with stellar wind.

Case AS - slow (nuclear-timescale) evolution to contact. For low mass ratios $\left(q_{0} \sim 1-1.5\right)$, and rather short periods $\left(0.8-2 \mathrm{~d}\right.$ at $\left.M_{1} \sim 4 M_{\odot}\right)$ the system misses contact during the thermal-timescale mass transfer. Then the increased mass of star 2 makes its evolution accelerate, ultimately faster than star 1's, and star 2 reaches its Roche lobe before star 1 has stopped filling its own. The system enters contact, but now with $q<1$ (typically $q \sim 0.4$ ). Some massive Otype binaries (e.g. TU Mus, Terrell, page 320) may be in this state although TU Mus is also a possible candidate for Case AR. Once again future evolution is uncertain: I suspect a merger, although there may be some other possibilities. Case AR would seem rather probable for the precursor binary of the F supergiant. Using an obvious notation where the components of the hypothetical precursor binary to star 2 are star 21 and star 22, we need $M_{21} \sim 4 M_{\odot}$, and the period to be about $2 \mathrm{~d}$, so that star 21 can evolve, at about the same rate as star 1 (the G supergiant), to near the terminal main sequence before initiating RLOF. That means that the companion would have to have been about $2 M_{\odot}$, and this puts the system in Case AR. Another but less likely possibility, I believe, is that star 21 was slightly less massive (say $3.5 M_{\odot}$ ) but also the period shorter $(\sim 1 \mathrm{~d})$, so that RLOF and a merger in Case AS could take place in the same time that star 1 has required to evolve to its present state.

Blue stragglers in clusters have often been suggested as due to binary interaction, with mergers being likely contributors. The F supergiant, I suggest, is in effect a blue straggler in the smallest of all possible clusters. There are probably not many ways of confirming that a particular star is or is not the result of a merger. FK Com stars, red giants that appear to be single but to have unusually rapid rotation and high activity that probably comes from the rotation, have been suggested as products of mergers in W UMa binaries (Webbink 1976; Bopp \& Stencel 1981). The F component of OW Gem does not however appear to be in unusually rapid rotation. This is a disappointment, but I will try to argue that the $F$ supergiant may have passed previously through a $G / K$ supergiant phase immediately after the merger, when unusual stellar activity took place and removed substantial angular momentum by stellar wind and magnetic braking. 
In fact the most likely way of recognising that a particular star is a merged remnant is to find it in a binary with a component that is less massive and yet more (or comparably) evolved: exactly as in OW Gem.

\section{V643 Ori}

V643 Ori is slightly similar to OW Gem in that we have two red giants with much more different masses than we would like ( $q \sim 0.6$ or 1.7 , depending on which component one believes was initially more massive). However most other circumstances are different: the period is short enough, the stars large enough, and the orbit circular enough that former RLOF is quite a possibility, although even the larger component only about half-fills its Roche lobe at present.

But former RLOF, at least of a standard variety, seems unlikely. The expected outcome would be either (a) a binary with a much more extreme mass ratio ( $q \sim 0.1$ is typical), or (b) a merged or very close remnant of common-envelope evolution, since star 1 would have had a deep convective envelope when it first filled its Roche lobe, and might be expected to have had dynamically unstable RLOF.

I suggest this is a product of a process called binary-enhanced stellar wind (Tout \& Eggleton 1988), but perhaps modified by another process that one might call partial accretion of stellar wind. Ignoring the latter as being potentially too complicated, let us suppose that the original binary had parameters $(3.5+$ $3.4 M_{\odot}, 120 \mathrm{~d}, e=0.3$ ). Star 1 would have approached close to, but not quite reached, its Roche lobe at helium ignition, in an orbit circularised by tidal friction to a period of $\sim 105 \mathrm{~d}$. In fact the period might have been reduced further, to say $80 \mathrm{~d}$, because quite a fraction of the orbital angular momentum would be turned into spin angular momentum of the large star 1 . Star 1 would be spun up to corotation, having previously spun down because of evolutionary expansion at least, and probably also some magnetic braking before tidal friction became important. A period of $80 \mathrm{~d}$ would be unusually rapid in such a large star, and so star 1 was probably highly active, as in many RS CVn binaries. The binary Z Her (Popper 1980; Tout \& Eggleton 1988; Popper 1988; Eggleton 2002) shows clear evidence that mass loss can be accelerated to be comparable to the nuclear timescale; in Z Her star 1, a red subgiant, is $20 \%$ less massive than star 2, though neither fills its Roche lobe. Whether a star can lose $\sim 50 \%$ of its mass by the same process remains to be seen.

The system is much complicated, however, by the possibility that both components have passed through helium ignition, and so there may have been two episodes of enhanced stellar wind, one from each star. It is then certainly not clear which star started out as the more massive (i.e. star 1). A lot depends on whether the angular-momentum loss associated with the mass loss is substantial or minimal. A lot also depends on what fraction of each wind might have been accreted by the other star. It would be difficult to firm up this speculation.

\section{Summary}

Even, and perhaps especially, for systems which are double-lined eclipsing binaries and which might therefore be supposed to have relatively well-determined 
parameters, there are some surprising examples to be found. One can hope that later redeterminations might remove anomalies. For Z Her the parameters changed very significantly between Popper (1980) and Popper (1988), although the mass anomaly actually increased. I believe it would not be good practice simply to ignore these anomalous systems in the hope that they will go away; but equally I believe it would not be good practice to build whole elaborate theories on three odd examples.

However there seem to me to be examples and concepts beyond these individual systems which make me support three modifications to standard wisdom:

(i) massive components $\left(\sim 10-30 M_{\odot}\right)$ that can be expected to experience RLOF in a moderately wide orbit of $\sim 50-250 \mathrm{~d}$ may actually experience rapid ejection of the entire envelope, with perhaps a small proportion of the wind accreted by the companion, and with rather little orbital shrinkage;

(ii) triples, and mergers of close binaries, may contribute significantly to the stellar population and its long-term evolution;

(iii) stellar wind in red giants may be enhanced very considerably, up to the nuclear timescale and perhaps even further, by the presence of close companions.

Acknowledgments. My visit has been supported financially by the DJEHUTY project at LLNL. Work performed at LLNL is supported by the DOE under contract W7405-ENG-48.

\section{References}

Batten, A. H., Fletcher, J. M., \& McCarthy, D. G. 1989, PDAO, 17, 1

Bopp, B. W., \& Stencel, R. E. 1981, ApJ, 247, L131

Eggleton, P. P. 2002, ApJ, in press

Gordon, K. D., Clayton, G. C., Smith, T. L., Aufdenberg, J. P., Drilling, J. S., Hanson, M. M., Anderson, C. M., \& Mulliss, C. L. 1998, AJ, 115, 2561

Griffin, R. F., \& Duquennoy, A. 1993, Obs, 113, 53

Hoffleit, D., \& Jaschek, C. 1983, The Bright Star Catalogue - 4th ed. (New Haven: Yale University Observatory)

Humphreys, R. M., \& Davidson, K. 1979, ApJ, 232, 409

Imbert, M. 1987, A\&AS, 71, 69

Lucy, L. B., \& Ricco, E. 1979, AJ, 84, 401

Mayer, P., Chochol, D., Drechsel, H., Lorenz, R., Plavec, M., Raja, T. \& Batten, A. H. 2001, A\&A, 375, 434

Nelson, C. A., \& Eggleton, P. P. 2001, ApJ, 552, 664

Popper, D. M. 1980, ARA\&A, 18, 115

Popper, D. M. 1988, AJ, 95, 1242

Tout, C. A., \& Eggleton, P. P. 1988, MNRAS, 231, 823

Webbink, R. F. 1976, ApJ, 209, 829 